\title{
Cardiac Autonomic Control in Relation to Other Prognostic Markers in Idiopathic Dilated Cardiomyopathy
}

\author{
Satu Kärkkäinen1,2, Ari Puhakka1, Jan Fedacko3, Jarkko Magga1,2, Kati Miettinen1, \\ Esko Vanninen4, Johanna Kuusisto1, Keijo J. Peuhkurinen1, Petri O. Tuomainen1,2 \\ ${ }^{1}$ Department of Internal Medicine, Kuopio University Hospital and University of Eastern Finland, Kuopio, Finland \\ ${ }^{2}$ Heart Center, Kuopio University Hospital and University of Eastern Finland, Kuopio, Finland \\ ${ }^{3}$ Center of Preventive and Sports Medicine, Safarik University and Pasteur Hospital, Kosice, Slovakia \\ ${ }^{4}$ Department of Clinical Physiology and Nuclear Medicine, Kuopio University Hospital and University of Eastern \\ Finland, Kuopio, Finland \\ Email: Petri.Tuomainen@uef.fi
}

Received 13 April 2015; accepted 17 May 2015; published 20 May 2015

Copyright (C) 2015 by authors and Scientific Research Publishing Inc.

This work is licensed under the Creative Commons Attribution International License (CC BY).

http://creativecommons.org/licenses/by/4.0/

(c) (i) Open Access

\begin{abstract}
Objectives: We set out to study cardiac autonomic tone in patients with idiopathic dilated cardiomyopathy (IDC), and whether it correlates with other established markers of disease progression and patient ultimate outcome. Design: Fifty-one IDC patients in sinus rhythm underwent extensive non-invasive and invasive evaluation during a three-day hospitalization period. The control group consisted of thirty-eight apparently healthy subjects who underwent 24-hour ambulatory ECG recording. Results: Heart rate variability (HRV) and heart rate turbulence (HRT) correlated with many previously established prognostic markers of IDC and congestive heart failure, especially measures of cardiorespiratory performance capacity and circulating neurohumoral factors $(p<$ 0.05 for all). Furthermore, attenuated HRV correlated with worse prognosis during a median follow-up of 6.8 years (range 5.1 - 8.1). Additionally, 24-hour time domain, low and high frequency components of frequency domain and non-linear HRV, excluding scaling exponents $(\alpha)$, were lower in IDC patients as compared with controls $(p<0.05$ for all); however, HRT was not significantly different. Conclusions: The magnitude of impairment in cardiac autonomic control correlates well with other prognostic markers of IDC, and is associated with unfavorable outcome.
\end{abstract}

\section{Keywords}

Heart Rate Variability, Heart Rate Turbulence, Cardiorespiratory Fitness, Vasoactive Peptides, Proinflammatory Cytokines 


\section{Introduction}

Dilated cardiomyopathy (DCM) is characterized by cardiac dilatation, reduced left ventricular systolic function and poor prognosis [1] [2]. A considerable proportion of DCM is of familial origin; however, infectious, inflammatory and immunological factors may play roles in the development and prognosis of the disease [3]-[6]. Functional class, cardiorespiratory fitness, and left ventricular function seem to be important prognostic markers in DCM of ischemic or idiopathic origin [7]-[9]. In addition, increased levels of vasoactive peptides, such as N-terminal pro-brain natriuretic peptide (NT-proBNP), and proinflammatory cytokines are linked to disease severity, but their prognostic significance has not been thoroughly studied in idiopathic dilated cardiomyopathy (IDC) [10]-[12].

Unfavorable changes in cardiac autonomic control, as measured by heart rate variability (HRV) and heart rate turbulence (HRT), occur in DCM independent of its etiology and the impairment correlates with prognosis [13]-[16]. Together with progressive worsening of congestive heart failure (CHF), major arrhythmic events resulting in sudden cardiac death (SCD) are a significant cause of mortality in patients with IDC. Impaired autonomic balance may predict malignant ventricular arrhythmias among this patient category; nevertheless, the findings have been controversial [17]-[19]. Similarly, markers like NT-proBNP may have predictive value in arrhythmogenesis [10] [20]. To approach the challenge of the individual prognostic evaluation, we set out to study the correlation of disturbances of cardiac autonomic control and other established markers of disease progression and patient outcome in IDC.

\section{Materials and Methods}

\subsection{Study Subjects and Protocol}

The study population consisted of fifty-one patients with IDC and sinus rhythm (SR). The patients were clinically well-characterized and living in the region of Kuopio University Hospital in eastern Finland. The diagnostic criteria for DCM were impaired left ventricular systolic function (ejection fraction, EF, $<45 \%$ ) and left ventricular dilatation (left ventricular end-diastolic diameter, LVEDD, $>27 \mathrm{~mm} / \mathrm{m}^{2}$ ) measured at the time of diagnosis, and no underlying diseases causing DCM [1].

The patients were hospitalized for a three-day study period, during which 24-hour ambulatory electrocardiography (ECG) registrations, laboratory testing, echocardiography, gated myocardial perfusion single photon emission computed tomography (SPECT), cardiopulmonary exercise testing and both-sided cardiac catheterization were carried out. Only patients without angiographic coronary artery disease were included in the study. All patients gave written informed consent, and the study was carried out in accordance with the Declaration of Helsinki, and was approved by the Ethics Committee of the University of Kuopio. The study design was prospective. After the initial three-day hospitalization period, all patients were followed regularly with yearly clinical visits up to four years. Thereafter, the clinical events were registered based on the medical records. None of the patients withdrew from the study.

The control group for the ambulatory ECG analyses consisted of thirty-eight consecutively recruited subjects who underwent elective 24-hour ECG registration and echocardiography due to symptomatic extrasystoles. All these subjects were otherwise apparently healthy with no other major cardiovascular symptoms, and had normal findings in echocardiography. However, ventricular ectopy was methodologically necessary to permit the determination of HRT. The controls did not differ statistically in mean age and gender distribution as compared with the patient group.

\subsection{Ambulatory ECG}

All the methods have been previously described in detail by Tuomainen et al. [21] [22]. In brief, ambulatory 24-hour ECG recordings were performed using digital recorders (Oxford Medilog Excel FD-3, Oxford Instruments Ltd., UK). The initial analysis of the ECG was performed using an automated procedure (Oxford Medilog Iris, Oxford Instruments Ltd., UK), and the reliability of the classified ECG templates were checked manually and corrected in appropriate cases. All sections containing other than pure sinus rhythm were excluded. Annotated RRI data was then transformed in ASCII-form and the final analyses were performed using custom software designed for a variety of time series (Win CPRS Version 1.156, Absolute Aliens Co., Finland).

HRT was determined by a signal averaged method from single ventricular premature complexes (VPCs) ful- 
filling the following two criteria: coupling interval within $80 \%$ and compensatory pause longer than $110 \%$ of the mean of the five preceding RR-intervals (RRIs). These five preceding and fifteen following RRIs had to be free of ectopic complexes. Two different parameters, turbulence onset (TO) and turbulence slope (TS) were computed. This method has been previously described by Schmidt et al. [23].

\subsection{Cardiopulmonary Exercise Test}

Cardiopulmonary exercise test was performed with a bicycle ergometer (Ergo-metrics 900, Ergo-line GmbH, Bitz, Germany) until exhaustion. The initial work load was $20 \mathrm{~W}$ with subsequent increments of $20 \mathrm{~W}$ per minute. Respiratory gas exchange was analyzed continuously with a computer-based system (Vmax 29, SensorMedics Corp., Yorba Linda, California, USA). The average values of oxygen uptake measured during the last 20 seconds of exercise were used for the maximal oxygen consumption $\left(\mathrm{VO}_{2 \max }\right)$.

\subsection{Cardiac Catheterization}

In the present study, all patients underwent left-sided and 96\% right-sided cardiac catheterization. Coronary angiography was performed in at least six projections with cranial and caudal angulated views and diameter stenoses of $\geq 50 \%$ were considered significant. Patients expressing even a single significant lesion in the main coronary branches were excluded from the study. Invasive pressures were measured from both sides. Venous blood samples for the $\mathrm{O}_{2}$-saturation determinations were taken from ten sites to exclude significant arteriovenous shunts and to determine cardiac output and index (CO, CI) by Fick's formula. An electrophysiological study (EPS) was performed to $82 \%$ of the patients. The stimulation sites were right ventricular apex (RVA) and right ventricular outflow tract (RVOT) employing identical stimulation protocols. The endpoint was predetermined as a combination of non-sustained ( $>15$ beats and $<30 \mathrm{sec}$ ) ventricular tachycardia (VT) or sustained ( $>30 \mathrm{sec}$ ) VT or ventricular fibrillation $(\mathrm{VF})$.

\subsection{Imaging Studies}

Left ventricular function and dimensions were measured at rest by ECG-gated SPECT and ${ }^{99 \mathrm{~m}}$ Tc-tetrofosmin (500 MBq; Myoview, Nycomed-Amersham plc, Buckinghamshire, England) and Quantitative Gated SPECT Application software (QGS v. 3.0, Cedars-Sinai Medical Center, Los Angeles, California, USA) was used.

\subsection{Vasoactive Peptide and Cytokine Analyses}

Blood samples for the determination of biomarkers reflecting the activation of neuroendocrine and inflammatory system were taken in standardized circumstances. The samples were stored at $-70^{\circ} \mathrm{C}$ until analyzed. All these methods have been previously described in detail [24] [25].

\subsection{Statistical Analysis}

The comparison of HRV and HRT parameters between the groups was performed by one-way analysis of variance (ANOVA) in the case of both normality and homogeneity of variances, otherwise a nonparametric Kruskal-Wallis test was used. Normality of the distribution of the variables was tested using one-sample Kolmogorov-Smirnov test. Crosstabulation and chi-square test were used when comparing the gender distribution of subjects between the groups. Correlation coefficients (Pearson's in cases of normality, otherwise Spearman's) between clinical measurements and HRV and HRT parameters were determined. Univariate logistic regression analysis was used for evaluating different prognostic factors. Due to the number of patients and endpoints, multivariate analysis was not considered in this study. Statistical significance (two-tailed tests) was set at 5\%. Statistical software used was SPSS for Windows, versions 13.0, 14.0 and 17.0 (Chicago, Ill., USA). The data are presented as means $\pm \mathrm{SD}$ (or $95 \% \mathrm{CI}$ ) or the number (\%) of subjects.

\section{Results}

\subsection{Study Subjects}

The groups of IDC patients and control subjects consisted of 37 and 26 men and 14 and 12 women, respectively. 
The mean age was 54 years (range 16 - 76) in the patient group and 52 years (range 21 - 74) in the control group ( $\mathrm{p}=\mathrm{ns}$ for both). In the patient group, the mean time from the primary diagnosis to the hospital study period was 2.5 years (range 0.1 - 9.7). Twenty (39\%) patients presented with left bundle branch block (LBBB). Twenty (39\%) patients were in New York Heart Association (NYHA) functional class I, 28 (55\%) patients in NYHA class II, and $3(6 \%)$ patients in NYHA class III, whereupon the patients had mild to moderate symptoms. Furthermore, the patients were adequately medicated (Table 1).

\subsection{HRV, HRT and Ectopic Beats in IDC Patients and Controls}

The mean count of supraventricular premature complexes was over ten fold in IDC patients as compared with controls $(p<0.01)$. The number of ventricular premature complexes did not differ significantly between the groups. The mean percentage of all edited beats in the whole registration was 5\% (95\% CI 3 - 8) in the patient group and $2 \%(95 \%$ CI 1 - 4) in the control group ( $p=n s)$. Neither did the mean RRI of the ambulatory 24-hour ECG registration differ significantly between the groups. The mean values of all time domain indices and the LF and HF components of frequency domain HRV were higher in the control group as compared with IDC patients ( $p<0.01$ for the time domain and $p<0.05$ for the frequency domain indices, respectively). The same was true also in the cases of nonlinear FD and the Poincaré plot ( $<<0.01$ for all). No significant difference in HRT was observed between the groups, however (Table 2).

\subsection{Correlation between HRV, HRT and Other Prognostic Factors in Patients with IDC}

In ergospirometry, the measures of cardiorespiratory fitness (Wlast4 and $\mathrm{VO}_{2 \mathrm{max}}$ ) correlated positively with HRV and TS and negatively with TO. Interestingly, HRV and HRT did not correlate with left ventricular enddiastolic diameter, volume or end-diastolic pressure; TO correlated only with LVEDD. On the other hand, HRV and TO correlated with left ventricular end-systolic diameter and ejection fraction. Furthermore, HRV and TS correlated directly, and TO inversely, with left ventricular diastolic function determined by E/A-ratio in echocardiography. Significant inverse correlations between HRV, TS and NT-proBNP resembled those between HRV, TS and PCWP. Similarly, HRV and TS correlated inversely, and TO directly, with the norepinephrine level. Furthermore, HRV and TS correlated inversely in a similar way with the proinflammatory cytokine IL-6 and hS-CRP. Neither HRV nor HRT correlated significantly with NT-proANP (Table 3, Figure 1).

\subsection{LBBB, Inducibility and Cardiac Autonomic Control}

IDC patients were divided into two subgroups according to the presence of LBBB in the 12-lead resting ECG. The mean values of all power spectral components, pNN50 and TS were lower and TO higher in the subgroup with LBBB as compared with the group without it ( $<0.05$ for all) (Table 4, Figure 2). Furthermore, patients were divided into two subgroups according to the inducibility in EPS, which was performed to 41 patients. Fifteen patients received the endpoint described earlier. The ratio of LF and HF power (mean \pm SD: $2.8 \pm 1.7$ vs. $4.6 \pm 2.4$ ), ln LF power (5.7 \pm 0.7 vs. $6.5 \pm 1.2)$ and TS (14.6 \pm 10.7 vs. $21.6 \pm 12.5$ ) were lower (p $<0.05$ for all) in the subgroup with inducible ventricular arrhythmias as compared with the group without malignant arrhythmias, respectively.

\subsection{Prognostic Significance of HRV and HRT}

Death, heart transplantation and implantation of an automatic cardioverter defibrillator and/or a biventricular pacemaker were predetermined as significant clinical endpoints. All the defibrillator implantations were based on clinical judgment and present guidelines. There were no prophylactic procedures. The biventricular pacemakers in this population were implanted in patients with progressive end-stage heart failure in spite of optimized medication. Biventricular pacemakers are known to improve the prognosis in these patients, and therefore the implantation of these devices was included in the combined endpoint cluster. Twelve (24\%) patients received an endpoint during a median follow-up period of 6.8 years (range 5.1 - 8.1). SDNN (mean \pm SD: $98 \pm 33$ vs. 133 $\pm 37, \mathrm{p}<0.01)$, Poincaré plot SD2 (137 \pm 47 vs. $186 \pm 52, \mathrm{p}<0.01)$, ln total power $(8.6 \pm 0.7$ vs. $9.2 \pm 0.6$, p < $0.05)$, $\ln U L F$ power (8.2 \pm 0.7 vs. $8.7 \pm 0.6, p<0.05)$ and $\ln \operatorname{VLF}$ power $(7.4 \pm 0.7$ vs. $7.9 \pm 0.7$, p < 0.05$)$ were lower in the group with endpoints as compared with the group without endpoints, respectively. HRT did not differ significantly between these two groups, however. 
Table 1. Characteristics of IDC patients.

Age (years)

Male/female

Years since diagnosis

Functional class (I/II/III, according to NYHA)

Medication

$\beta$-receptor blockers

Angiotensin converting enzyme inhibitors

Angiotensin-receptor blockers

Diuretics

Spironolactone

Digitalis

Warfarin

Acetosalicylic acid

Statins

Long-acting nitrates

Calcium-channel blockers

Other antiarrhythmic drug

BMI $\left(\mathrm{kg} / \mathrm{m}^{2}\right)$

SBP (mmHg)

DBP (mmHg)

LBBB

Ergospirometry

Wlast4 (W)

$\mathrm{VO}_{2 \max }(\mathrm{ml} / \mathrm{min} / \mathrm{kg})$

\section{Gated SPECT}

LVEDV (ml)

LVESV (ml)

LVEF (\%)

\section{Catheterization}

LVEDP (mmHg)

PAPm (mmHg)

PCWPm (mmHg)

CI $\left(1 / \mathrm{min} / \mathrm{m}^{2}\right)$

\section{Clinical chemistry}

$\mathrm{NE}(\mathrm{nmol} / \mathrm{l})$

NT-proBNP (pmol/l)

hs-CRP (mg/l)

IL-6 (pg/ml)

Echocardiography

$\begin{array}{lc}\operatorname{LVEDD}(\mathrm{mm}) & 65 \pm 9 \\ \operatorname{LVESD}(\mathrm{mm}) & 52 \pm 10 \\ \operatorname{LVEF}(\%) & 38 \pm 9 \\ \text { E/A-ratio } & 1.0 \pm 0.6\end{array}$

$54 \pm 13$

37 (73\%)/14 (27\%)

$2.5 \pm 2.8$

20 (39\%)/28 (55\%)/3 (6\%)

47 (92\%)

$41(80 \%)$

6 (12\%)

16 (31\%)

1 (2\%)

10 (20\%)

17 (33\%)

14 (27\%)

11 (22\%)

4 (8\%)

1 (2\%)

1 (2\%)

$28 \pm 5$

$129 \pm 22$

$82 \pm 12$

20 (39\%)

$123 \pm 51$

$21.5 \pm 8.5$

$172 \pm 90$

$126 \pm 88$

$30 \pm 12$

$15 \pm 8$

$18 \pm 7$

$10 \pm 6$

$2.9 \pm 1.9$

$2.3 \pm 1.2$

$179 \pm 183$

$2.8 \pm 3.4$

$2.4 \pm 1.6$

$65 \pm 9$

$52 \pm 10$

$1.0 \pm 0.6$

Data are presented as mean \pm SD or number (\%) of patients. BMI: body mass index; CI: cardiac index; DBP: diastolic blood pressure; E/A-ratio: the ratio of maximal velocities of diastolic E- and A-waves; ECG: electrocardiogram; hs-CRP: high-sensitivity C-reactive protein; IDC: idiopathic dilated cardiomyopathy; IL-6: interleukin-6; LBBB: left bundle branch block; LVEDD: left ventricular end-diastolic diameter; LVEDP: left ventricular end-diastolic pressure; LVEDV: left ventricular end-diastolic volume; LVEF: left ventricular ejection fraction; LVESD: left ventricular end-systolic diameter; LVESV: left ventricular end-systolic volume; NE: norepinephrine; NT-proBNP: N-terminal pro-brain natriuretic peptide; NYHA: New York Heart Association; PAPm: mean pulmonary arterial pressure; PCWPm: mean pulmonary capillary wedge pressure; SBP: systolic blood pressure; SPECT: single photon emission computed tomography; $\mathrm{VO}_{2 \max }$ : maximal oxygen consumption; Wlast4: the mean load of the last 4 minutes of exercise. 
Table 2. Ambulatory 24-hour ECG registration parameters in healthy controls and in IDC patients.

\begin{tabular}{|c|c|c|c|}
\hline & Controls $(\mathrm{n}=38)$ & IDC patients $(n=51)$ & $\mathrm{p}$ \\
\hline VPC count & $1448 \pm 2929$ & $2638 \pm 5258$ & ns \\
\hline SVPC count & $54 \pm 221$ & $633 \pm 2504$ & $<0.01$ \\
\hline Mean RRI (ms) & $916 \pm 150$ & $877 \pm 117$ & ns \\
\hline SDNN (ms) & $153 \pm 44$ & $124 \pm 39$ & $<0.01$ \\
\hline RMSSD (ms) & $41 \pm 29$ & $30 \pm 19$ & $<0.01$ \\
\hline PNN50 (\%) & $12.6 \pm 10.5$ & $7.2 \pm 8.0$ & $<0.01$ \\
\hline FD & $1.65 \pm 0.07$ & $1.59 \pm 0.09$ & $<0.01$ \\
\hline SD 1 (Poincare plot) & $29 \pm 20$ & $21 \pm 13$ & $<0.01$ \\
\hline SD 2 (Poincare plot) & $214 \pm 61$ & $174 \pm 54$ & $<0.01$ \\
\hline$\alpha 1$ & $1.03 \pm 0.02$ & $1.03 \pm 0.01$ & ns \\
\hline$\alpha 2$ & $0.98 \pm 0.01$ & $0.98 \pm 0.02$ & ns \\
\hline Total power (ln) & $9.3 \pm 0.6$ & $9.1 \pm 0.7$ & ns \\
\hline ULF power (ln) & $8.9 \pm 0.6$ & $8.6 \pm 0.7$ & ns \\
\hline VLF power (ln) & $8.0 \pm 0.6$ & $7.8 \pm 0.7$ & ns \\
\hline LF power (ln) & $6.5 \pm 0.9$ & $6.1 \pm 1.2$ & $<0.05$ \\
\hline HF power (ln) & $5.4 \pm 1.0$ & $4.9 \pm 1.2$ & $<0.05$ \\
\hline LF/HF ratio & $3.6 \pm 2.2$ & $3.7 \pm 2.2$ & ns \\
\hline TO (\%) & $-3.2 \pm 4.6$ & $-3.4 \pm 4.4$ & ns \\
\hline TS (ms/RRI) & $22.6 \pm 17.6$ & $17.7 \pm 12.1$ & ns \\
\hline
\end{tabular}

Data are presented as mean \pm SD. $\alpha$ 1: short-term scaling exponent alpha; $\alpha$ 2: long-term scaling exponent alpha; ECG: electrocardiogram; FD: fractal dimension; HF: high frequency; IDC: idiopathic dilated cardiomyopathy; LF: low frequency; PNN50: percentage of RRIs differing more than $50 \mathrm{~ms}$ from the previous RRI; RMSSD: root mean square of successive difference of normal RRIs; RRI: RR-interval; SD: standard deviation; SDNN: standard deviation of normal RRIs; SVPC: supraventricular premature complex; TO: turbulence onset; TS: turbulence slope; ULF: ultra low frequency; VLF: very low frequency; VPC: ventricular premature complex.
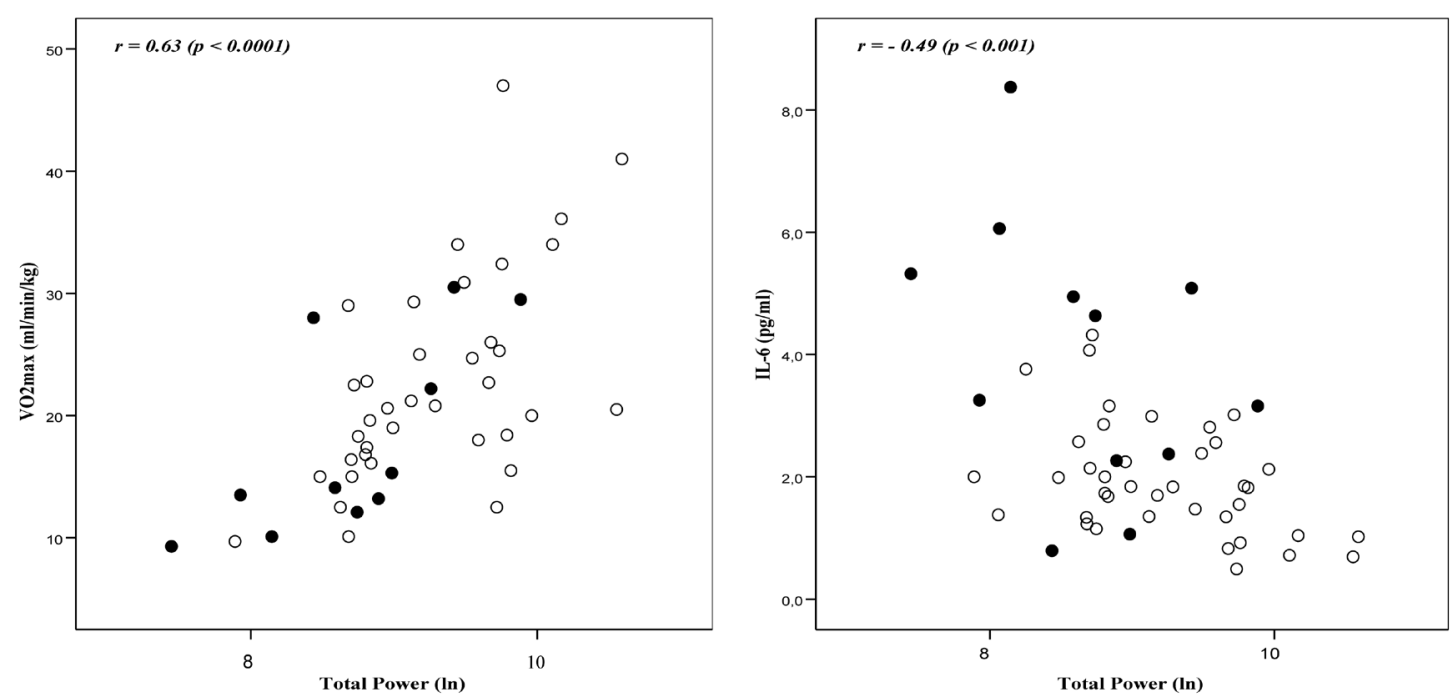

Figure 1. Correlations between total power and maximal oxygen consumption $\left(\mathrm{VO}_{2 \max }\right)$ and interleukin 6 (IL-6). Closed circles: patients with clinical endpoints; open circles: patients without clinical endpoints. 
Table 3. Correlation coefficients between HRV, HRT and clinical parameters in IDC patients.

\begin{tabular}{|c|c|c|c|c|c|c|c|}
\hline & TP & LF & $\mathrm{HF}$ & SD1 & SD2 & TO & TS \\
\hline \multicolumn{8}{|c|}{ Ergospirometry } \\
\hline Wlast4 & $0.56^{* * * *}$ & $0.61^{* * * *}$ & $0.40^{* *}$ & $0.40^{* *}$ & $0.46^{* *}$ & $-0.41^{* *}$ & $0.53 * * *$ \\
\hline $\mathrm{VO}_{2 \max }$ & $0.63^{* * * *}$ & $0.60^{* * * *}$ & $0.47^{* * *}$ & $0.44^{* *}$ & $0.48^{* * *}$ & $-0.40^{* *}$ & $0.59 * * * *$ \\
\hline \multicolumn{8}{|l|}{ Gated SPECT } \\
\hline LVEDV & -0.15 & -0.14 & -0.21 & -0.14 & -0.14 & 0.28 & -0.08 \\
\hline LVESV & -0.22 & -0.18 & -0.25 & -0.18 & -0.17 & $0.36^{*}$ & -0.15 \\
\hline LVEF & $0.34^{*}$ & 0.22 & 0.25 & 0.18 & $0.30 *$ & $-0.33^{*}$ & 0.22 \\
\hline \multicolumn{8}{|c|}{ Catheterization } \\
\hline LVEDP & -0.06 & -0.01 & -0.07 & 0.00 & -0.10 & -0.04 & -0.15 \\
\hline PAPm & -0.13 & -0.24 & -0.13 & $-0.30^{*}$ & -0.13 & 0.13 & $-0.40^{* *}$ \\
\hline PCWPm & $-0.32 *$ & $-0.36^{*}$ & $-0.38 * *$ & $-0.38 * *$ & $-0.40 * *$ & 0.10 & $-0.43^{* *}$ \\
\hline CI & -0.01 & -0.10 & -0.02 & -0.13 & -0.06 & -0.07 & -0.12 \\
\hline \multicolumn{8}{|c|}{ Clinical chemistry } \\
\hline $\mathrm{NE}$ & $-0.35^{*}$ & $-0.31^{*}$ & $-0.30 *$ & $-0.33^{*}$ & $-0.34 *$ & $0.33^{*}$ & $-0.28 *$ \\
\hline NT-proANP & -0.25 & -0.25 & -0.26 & -0.14 & -0.24 & 0.17 & -0.26 \\
\hline NT-proBNP & $-0.40^{* *}$ & $-0.38^{* *}$ & -0.29 & -0.27 & $-0.38^{* *}$ & 0.22 & $-0.41 * *$ \\
\hline hs-CRP & $-0.47 * * *$ & $-0.34^{*}$ & $-0.37 * *$ & $-0.43^{* *}$ & $-0.47 * * *$ & 0.02 & $-0.29 *$ \\
\hline IL-6 & $-0.49 * * *$ & $-0.39 * *$ & $-0.34 *$ & $-0.38^{* *}$ & $-0.50^{* * *}$ & 0.06 & $-0.37 * *$ \\
\hline \multicolumn{8}{|c|}{ Echocardiography } \\
\hline LVEDD & -0.20 & -0.19 & -0.21 & -0.18 & -0.17 & $0.33^{*}$ & -0.09 \\
\hline LVESD & $-0.30 *$ & -0.22 & -0.26 & -0.25 & $-0.29 *$ & $0.28 *$ & -0.12 \\
\hline LVEF & $0.38^{* *}$ & 0.26 & $0.32 *$ & $0.34 *$ & $0.36^{*}$ & -0.26 & 0.21 \\
\hline E/A-ratio & $0.35 *$ & $0.36^{*}$ & 0.28 & $0.37 *$ & $0.36 *$ & $-0.47 * *$ & $0.42 * *$ \\
\hline
\end{tabular}

${ }^{*} \mathrm{p}<0.05,{ }^{* *} \mathrm{p}<0.01,{ }^{* * *} \mathrm{p}<0.001$ and $* * * * \mathrm{p}<0.0001$. CI: cardiac index; E/A-ratio: the ratio of maximal velocities of diastolic E- and A-waves; HF: high frequency power; HRT: heart rate turbulence; HRV: heart rate variability; hs-CRP: high-sensitivity C-reactive protein; IDC: idiopathic dilated cardiomyopathy; IL-6: interleukin-6; LF: low frequency power; LVEDD: left ventricular end-diastolic diameter; LVEDP: left ventricular end-diastolic pressure; LVEDV: left ventricular end-diastolic volume; LVEF: left ventricular ejection fraction; LVESD: left ventricular end-systolic diameter; LVESV: left ventricular end-systolic volume; NE: norepinephrine; NT-proANP: N-terminal pro-atrial natriuretic peptide; NT-proBNP: $\mathrm{N}$-terminal pro-brain natriuretic peptide; PAPm: mean pulmonary arterial pressure; PCWPm: mean pulmonary capillary wedge pressure; SD: standard deviation; SPECT: single photon emission computed tomography; TO: turbulence onset; TP: total power; TS: turbulence slope; $\mathrm{VO}_{2 \text { max }}$ maximal oxygen consumption; Wlast4: the mean load of the last 4 minutes of exercise.
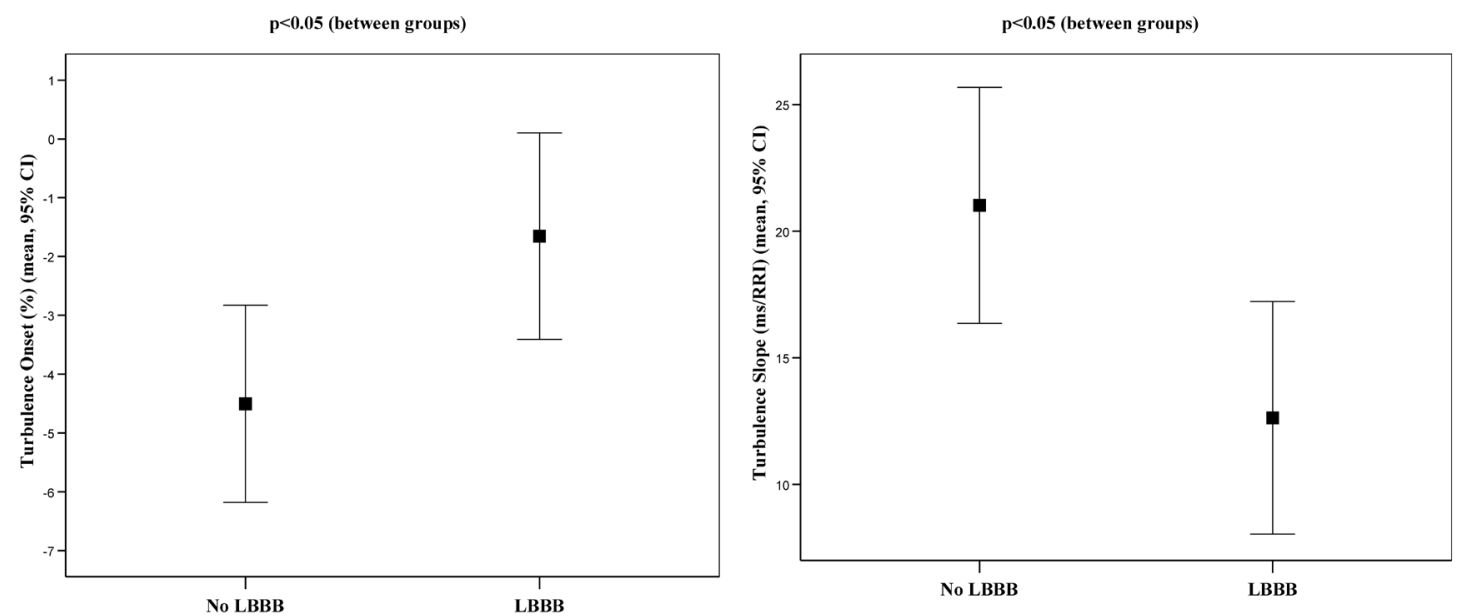

Figure 2. Comparison of heart rate turbulence according to the presence of left bundle branch block (LBBB). Data are presented as mean (95\% CI). 
Table 4. HRV and HRT parameters in IDC patients with and without LBBB.

\begin{tabular}{|c|c|c|c|}
\hline & $\operatorname{LBBB}(n=20)$ & No LBBB $(\mathrm{n}=31)$ & $\mathrm{p}$ \\
\hline Mean RRI (ms) & $890 \pm 108$ & $869 \pm 124$ & ns \\
\hline SDNN (ms) & $111 \pm 35$ & $133 \pm 39$ & 0.051 \\
\hline RMSSD (ms) & $24 \pm 9$ & $34 \pm 22$ & 0.070 \\
\hline PNN50 (\%) & $3.6 \pm 2.7$ & $9.6 \pm 9.3$ & $<0.05$ \\
\hline FD & $1.58 \pm 0.07$ & $1.60 \pm 0.10$ & ns \\
\hline SD 1 (Poincare plot) & $17 \pm 6$ & $24 \pm 16$ & 0.057 \\
\hline SD 2 (Poincare plot) & $156 \pm 50$ & $186 \pm 55$ & 0.056 \\
\hline$\alpha 1$ & $1.03 \pm 0.01$ & $1.03 \pm 0.01$ & ns \\
\hline$\alpha 2$ & $0.98 \pm 0.02$ & $0.98 \pm 0.02$ & ns \\
\hline Total power (ln) & $8.8 \pm 0.7$ & $9.3 \pm 0.7$ & $<0.05$ \\
\hline ULF power (ln) & $8.4 \pm 0.7$ & $8.8 \pm 0.6$ & $<0.05$ \\
\hline VLF power (ln) & $7.5 \pm 0.7$ & $7.9 \pm 0.7$ & $<0.05$ \\
\hline LF power (ln) & $5.6 \pm 0.8$ & $6.4 \pm 1.2$ & $<0.05$ \\
\hline HF power (ln) & $4.5 \pm 0.8$ & $5.2 \pm 1.3$ & $<0.05$ \\
\hline $\mathrm{LF} / \mathrm{HF}$ ratio & $3.4 \pm 2.1$ & $3.9 \pm 2.2$ & ns \\
\hline TO (\%) & $-1.7 \pm 3.7$ & $-4.5 \pm 4.5$ & $<0.05$ \\
\hline TS (ms/RRI) & $12.6 \pm 9.8$ & $21.0 \pm 12.5$ & $<0.05$ \\
\hline
\end{tabular}

Data are presented as mean \pm SD. $\alpha$ 1: short-term scaling exponent alpha; $\alpha$ 2: long-term scaling exponent alpha; ECG: electrocardiogram; FD: fractal dimension; HF: high frequency; IDC: idiopathic dilated cardiomyopathy; LF: low frequency; PNN50: percentage of RRIs differing more than $50 \mathrm{~ms}$ from the previous RRI; RMSSD: root mean square of successive difference of normal RRIs; RRI: RR-interval; SD: standard deviation; SDNN: standard deviation of normal RRIs; TO: turbulence onset; TS: turbulence slope; ULF: ultra low frequency; VLF: very low frequency.

In univariate logistic regression analysis, mean RRI (OR 0.993; 95\% CI $0.987-0.999, \mathrm{p}=0.046$ ), SDNN (OR 0.966; CI 0.940 - 0.993, $\mathrm{p}=0.014$ ), SD2 (OR 0.975; CI 0.956 - 0.995, $\mathrm{p}=0.013$ ), ln total power (OR 0.247; CI 0.077 - 0.796, $\mathrm{p}=0.019$ ), ln ULF power (OR 0.236; CI 0.071 - 0.783, $\mathrm{p}=0.018$ ), ln VLF power (OR 0.349; CI 0.123 - 0.991, $\mathrm{p}=0.048$ ), PCWP (OR 1.097; CI 1.011 - 1.190, $\mathrm{p}=0.025)$, NT-proBNP (OR 1.003; CI 1.001 - 1.006, $\mathrm{p}=0.003$ ), LVEDD (OR 1.086; CI 1.026 - 1.149, $\mathrm{p}=0.004$ ), LVESD (OR 1.099; CI 1.040 - 1.161, $\mathrm{p}<$ 0.001), LVEDV (OR 1.010; CI 1.003 - 1.016, p = 0.004), LVESV (OR 1.013; CI 1.005 - 1.022, p = 0.001), and LVEF (OR 0.919; CI $0.873-0.968, \mathrm{p}=0.001$ ) were significant predictors of patient outcome (Table 5). However, the number of patients and endpoints were considered insufficient for adequate multivariate analysis.

\section{Discussion}

Recently, the GISSI-HF trial reported that on top of other established determinants, HRT and low-frequency HRV had an incremental value in predicting both "pump failure" and arrhythmogenic mortality in NYHA III IV HF patients at a median follow-up of four years [26]. In the present study, HRV and HRT were associated with many other previously established prognostic markers (i.e. ventricular dimensions and function, NYHA class, LBBB, age, natriuretic peptides, ventricular arrhythmias, atrial fibrillation, markers of fibrosis, and left atrial volume) of CHF in IDC patients; attenuated HRV was further associated with impaired prognosis at a median follow-up of 6.8 years (range 5.1 - 8.1) [7] [17] [20] [27]-[32]. Therefore, it seems that both HRV and HRT are valuable in the long-term risk assessment, not only in patients with severe, but also in those with milder HF. One of the strengths of the present study is that it was carried out in a well-defined and thoroughly studied group of patients with DCM, with proper follow-up. These patients clearly demonstrated increased LV dimensions and decreased LV systolic function in conjunction with both subjective and objective attenuation of cardiorespiratory exercise capacity, as well as increased level of NT-proBNP (Table 1). Control subjects underwent echocardiography to exclude clinically significant structural and functional myocardial abnormalities. 
Table 5. Univariate logistic regression analysis for prognostic factors in IDC..

\begin{tabular}{|c|c|c|c|c|}
\hline Factor & OR & $95 \%$ CI & Wald index & $\mathrm{p}$ value \\
\hline Mean RRI & 0.993 & $0.987-0.999$ & 4.0 & 0.046 \\
\hline SDNN & 0.966 & $0.940-0.993$ & 6.1 & 0.014 \\
\hline SD2 (Poincare plot) & 0.975 & $0.956-0.995$ & 6.2 & 0.013 \\
\hline Total power (ln) & 0.247 & $0.077-0.796$ & 5.5 & 0.019 \\
\hline ULF power (ln) & 0.236 & $0.071-0.783$ & 5.6 & 0.018 \\
\hline VLF power (ln) & 0.349 & $0.123-0.991$ & 3.9 & 0.048 \\
\hline LVEDD (M-m Echo) & 1.086 & $1.026-1.149$ & 8.1 & 0.004 \\
\hline LVESD (M-m Echo) & 1.099 & $1.040-1.161$ & 11.2 & $<0.001$ \\
\hline LVEF (M-m Echo) & 0.919 & $0.873-0.967$ & 10.6 & $<0.001$ \\
\hline LVEDV (2D Echo) & 1.010 & $1.002-1.019$ & 6.3 & 0.012 \\
\hline LVESV (2D Echo) & 1.014 & $1.004-1.024$ & 7.9 & 0.005 \\
\hline LVEF (2D Echo) & 0.929 & $0.880-0.980$ & 7.2 & 0.007 \\
\hline LVEDV (SPECT) & 1.010 & $1.003-1.016$ & 8.3 & 0.004 \\
\hline LVESV (SPECT) & 1.013 & $1.005-1.022$ & 10.3 & 0.001 \\
\hline LVEF (SPECT) & 0.919 & $0.873-0.968$ & 10.3 & 0.001 \\
\hline PCWP & 1.097 & $1.011-1.190$ & 5.0 & 0.025 \\
\hline NT-proBNP & 1.003 & $1.001-1.006$ & 8.8 & 0.003 \\
\hline
\end{tabular}

RRI = RR-interval; SDNN = standard deviation of normal RRIs; SD = standard deviation; ULF = ultra low frequency; VLF = very low frequency; LVEDD = left ventricular end-diastolic diameter; M-m = M-mode; LVESD = left ventricular end-systolic diameter; LVEF = left ventricular ejection fraction; LVEDV = left ventricular end-diastolic volume; LVESV = left ventricular end-systolic volume; SPECT = single photon emission computed tomography; PCWP = pulmonary capillary wedge pressure; NT-proBNP = N-terminal pro-brain natriuretic peptide.

During the era before HRT, DCM patients were reported to have lower time-domain HRV values as compared with healthy controls. Moreover, low HRV was associated with NYHA class, LVEDD, LVEF and $\mathrm{VO}_{2 \max }$. HRV was also associated with patient outcome at two-year follow-up [15]. In our study, 24-hour time-domain, LF and HF components of frequency-domain, and non-linear HRV indices, excluding scaling exponents $(\alpha)$, were prominently lower in DCM patients as compared with controls. Our results not only agree with the study of Yi et al. [15], but also indicate that deteriorated HRV may have prognostic value in mild to moderate IDC. However, in the present study, HRT did not differ significantly between IDC patients and controls. Interestingly, in the GISSI-HF trial, HRT (TS) had an incremental value in arrhythmia prediction only in the subgroup of patients with EF $>30 \%$ [26]. Attenuated cardiac autonomic nervous function was previously shown to predict malignant ventricular arrhythmias and SCD in IDC patients [18] [19]. In another study of patients with DCM treated with implanted cardioverter defibrillator, adverse outcomes were associated with NYHA functional class and renal function [32]. In our study, HRV and HRT were widely associated with well-known markers of left ventricular dysfunction and CHF. Furthermore, our patients with inducible VT/VF in EPS expressed significantly lower TS and low-frequency HRV as compared with patients with no inducible arrhythmias. These observations are in line with the GISSI-HF trial.

LBBB is associated with increased mortality in CHF patients, especially those with atrial fibrillation (AF) [27]. However, HRV and HRT cannot be determined among patients with AF. Our results confirm that in patients with SR, LBBB is strongly associated with deteriorated autonomic regulation; both are markers of impaired prognosis. In previous studies, the inducibility of malignant ventricular tachyarrhythmias in EPS had no significant value in predicting the risk of severe arrhythmias and SCD [33] [34]. Nevertheless, the results of the present study concerning the association between inducibility and autonomic nervous indices should be interpreted with caution; it may indicate that inducibility might well be associated with impaired autonomic balance, 
and possibly with the risk of spontaneous ventricular arrhythmias.

The patients in this study were adequately medicated; $92 \%$ of them taking $\beta$-blockers and ACE-inhibitors or AT-receptor blockers. It is noteworthy that many of them had improved clinically and showed increases in LVEF after the time point of primary diagnosis, two to five years prior to the current study. According to the guidelines, $\beta$-blockers and blockers of the renin-angiotensin-aldosterone system form the basis of medical treatment in patients with systolic dysfunction [35]. Effective and long-lasting treatment with these agents probably has an important protective role in DCM. Furthermore, both $\beta$-blockers and ACE-inhibitors have favorable effects on vagal control and HRV [36] [37]. Thus, in our study, the differences in HRV parameters between the two study groups would have been even more prominent should ambulatory ECG recording was performed before the start of treatment.

The results of the present study suggest a more preserved cardiac autonomic nervous tone in IDC patients with better exercise capacity. The results also suggest that impaired cardiac autonomic control is linked not only to depressed systolic function, but also diastolic function. Our findings also show that autonomic nervous indices and elevated left atrial filling pressure are tightly coupled. The association between cardiac autonomic control, on one hand, and filling pressures, natriuretic peptides, norepinephrine and inflammatory markers on the other hand, can be considered as multidimensional in nature. When elevated, natriuretic peptides and filling pressures reflect increased cardiac wall stress [38]-[40]. Furthermore, the increased cardiac load and stretch affect afferent impulses in the autonomic nervous system. HRV and the baroreflex sensitivity are documented to improve with cardiac resynchronization therapy. This is suggested to be due to deactivation of cardiac sympathetic afferent reflex [41] [42], the chronic activation of which being considered as a crucial pathophysiological mechanism in CHF.

Regarding the catecholamine response in our study, attenuated HRV and HRT may reflect the compensatory neurohumoral activation and increased sympathetic tone. Moreover, it has been suggested that inflammatory processes play a pivotal role in DCM and might even be involved in autonomic denervation in CHF, but the findings have remained controversial [43]-[45]. We found significant inverse correlations between autonomic nervous indices and hs-CRP and IL-6. Interestingly, in animal models, myocardial infarction-related damage induces proinflammatory cytokine synthesis not only in the heart but also in the brain. This is suggested to be due to cardiac sympathetic afferent activation. On the other hand, an intact vagus nerve seems to be required for the full expression of proinflammatory cytokines in damaged myocardium and further in blood circulation [46]. Although there seems to be an association between the autonomic nervous and inflammatory markers in IDC, the correlations are still far from clear.

\section{Limitations}

First of all, the number of IDC patients in the present study was limited due to the prevalence of SR. Secondly, the incidence of endpoints was relatively small and statistically insufficient for proper multivariate regression analyses. It is noteworthy, that IDC is a progressive disease, and the clinical course can change over time, as well as with changes in the type and dosage of medications. Thus, the cross-sectional measurements of different clinical parameters may limit the evaluation of the long-term prognosis. Basically, the aim of the present study was not to assess the relative value of HRV and HRT versus other established prognostic indicators of idiopathic DCM. This aspect clearly warrants more investigation with larger patient populations.

\section{Conclusion}

Impaired cardiac autonomic control correlates with other clinical and prognostic markers in patients of IDC, and associates with patient outcome. The significance of cardiac autonomic tone impairment and other established measures of disease progression and patient outcome still need further larger-scale studies.

\section{Acknowledgements}

We wish to express our thanks to our study nurse, Ms Satu Nenonen, for her valuable assistance throughout the study. This study was supported by grants from the Finnish Foundation for Cardiovascular Research, the Finnish Foundation for Medicine, the Finnish Cultural Foundation, North Savo Regional fund and the research funds of Kuopio University Hospital, Finland. 


\section{Conflict of Interest}

On behalf of all authors, the corresponding author states that there is no conflict of interest.

\section{References}

[1] Manolio, T.A., Baughman, K.L., Rodeheffer, R., Pearson, T.A., Bristow, J.D., Michels, V.V., et al. (1992) Prevalence and Etiology of Idiopathic Dilated Cardiomyopathy (Summary of a National Heart, Lung, and Blood Institute Workshop). American Journal of Cardiology, 69, 1458-1466. http://dx.doi.org/10.1016/0002-9149(92)90901-A

[2] Richardson, P.J. (1996) Assessment of Myocardial Damage in Dilated Cardiomyopathy. European Heart Journal, 17, 489-490. http://dx.doi.org/10.1093/oxfordjournals.eurheartj.a014894

[3] Martino, T.A., Liu, P. and Sole, M.J. (1994) Viral Infection and the Pathogenesis of Dilated Cardiomyopathy. Circulation Research, 74, 182-188. http://dx.doi.org/10.1161/01.RES.74.2.182

[4] Mestroni, L., Krajinovic, M., Severini, G.M., Milasin, J., Pinamonti, B., Rocco, C., et al. (1995) Molecular Genetics of Dilated Cardiomyopathies. European Heart Journal, 16, 5-9. http://dx.doi.org/10.1093/eurheartj/16.suppl_O.5

[5] Calabrese, F. and Thiene, G. (2003) Myocarditis and Inflammatory Cardiomyopathy: Microbiological and Molecular Biological Aspects. Cardiovascular Research, 60, 11-25. http://dx.doi.org/10.1016/S0008-6363(03)00475-9

[6] Noutsias, M., Pauschinger, M., Poller, W.C., Schultheiss, H.P. and Kuhl, U. (2003) Current Insights into the Pathogenesis, Diagnosis and Therapy of Inflammatory Cardiomyopathy. Heart Failure Monitoring, 3, 127-135.

[7] van den Broek, S.A., van Veldhuisen, D.J., de Graeff, P.A., Landsman, M.L., Hillege, H. and Lie, K.I. (1992) Comparison between New York Heart Association Classification and Peak Oxygen Consumption in the Assessment of Functional Status and Prognosis in Patients with Mild to Moderate Chronic Congestive Heart Failure Secondary to Either Ischemic or Idiopathic Dilated Cardiomyopathy. American Journal of Cardiology, 70, 359-363. http://dx.doi.org/10.1016/0002-9149(92)90619-A

[8] Cicoira, M., Zanolla, L., Latina, L., Rossi, A., Golia, G., Brighetti, G., et al. (2001) Frequency, Prognosis and Predictors of Improvement of Systolic Left Ventricular Function in Patients with "Classical” Clinical Diagnosis of Idiopathic Dilated Cardiomyopathy. European Journal of Heart Failure, 3, 323-330. http://dx.doi.org/10.1016/S1388-9842(00)00150-1

[9] Faris, R., Henein, M.Y. and Coats, A.J. (2003) Ventricular Long Axis Function Is Predictive of Outcome in Patients with Chronic Heart Failure Secondary to Non-Ischemic Dilated Cardiomyopathy. Medical Science Monitor, 9, CR456CR465.

[10] Simon, T., Becker, R., Voss, F., Bikou, O., Hauck, M., Licka, M., et al. (2008) Elevated B-Type Natriuretic Peptide Levels in Patients with Nonischemic Cardiomyopathy Predict Occurrence of Arrhythmic Events. Clinical Research in Cardiology, 97, 306-309. http://dx.doi.org/10.1007/s00392-007-0629-2

[11] Munger, M.A., Johnson, B., Amber, I.J., Callahan, K.S. and Gilbert, E.M. (1996) Circulating Concentrations of Proinflammatory Cytokines in Mild or Moderate Heart Failure Secondary to Ischemic or Idiopathic Dilated Cardiomyopathy. American Journal of Cardiology, 77, 723-727. http://dx.doi.org/10.1016/S0002-9149(97)89206-5

[12] Aukrust, P., Ueland, T., Lien, E., Bendtzen, K., Muller, F., Andreassen, A.K., et al. (1999) Cytokine Network in Congestive Heart Failure Secondary to Ischemic or Idiopathic Dilated Cardiomyopathy. American Journal of Cardiology, 83, 376-382. http://dx.doi.org/10.1016/S0002-9149(98)00872-8

[13] Voss, A., Baier, V., Schumann, A., Hasart, A., Reinsperger, F., Schirdewan, A., et al. (2002) Postextrasystolic Regulation Patterns of Blood Pressure and Heart Rate in Patients with Idiopathic Dilated Cardiomyopathy. The Journal of Physiology, 538, 271-278. http://dx.doi.org/10.1113/jphysiol.2001.013044

[14] Carvajal, R., Wessel, N., Vallverdu, M., Caminal, P. and Voss, A. (2005) Correlation Dimension Analysis of Heart Rate Variability in Patients with Dilated Cardiomyopathy. Computer Methods and Programs in Biomedicine, 78, 133140. http://dx.doi.org/10.1016/j.cmpb.2005.01.004

[15] Yi, G., Goldman, J.H., Keeling, P.J., Reardon, M., McKenna, W.J. and Malik, M. (1997) Heart Rate Variability in Idiopathic Dilated Cardiomyopathy: Relation to Disease Severity and Prognosis. Heart, 77, 108-114. http://dx.doi.org/10.1136/hrt.77.2.108

[16] Smilde, T.D., van Veldhuisen, D.J. and van den Berg, M.P. (2009) Prognostic Value of Heart Rate Variability and Ventricular Arrhythmias during 13-Year Follow-Up in Patients with Mild to Moderate Heart Failure. Clinical Research in Cardiology, 98, 233-239. http://dx.doi.org/10.1007/s00392-009-0747-0

[17] Grimm, W., Christ, M., Bach, J., Muller, H.H. and Maisch, B. (2003) Noninvasive Arrhythmia Risk Stratification in Idiopathic Dilated Cardiomyopathy: Results of the Marburg Cardiomyopathy Study. Circulation, 108, 2883-2891. http://dx.doi.org/10.1161/01.CIR.0000100721.52503.85

[18] Fauchier, L., Babuty, D., Cosnay, P. and Fauchier, J.P. (1999) Prognostic Value of Heart Rate Variability for Sudden 
Death and Major Arrhythmic Events in Patients with Idiopathic Dilated Cardiomyopathy. Journal of the American College of Cardiology, 33, 1203-1207. http://dx.doi.org/10.1016/S0735-1097(99)00021-2

[19] Rashba, E.J., Estes, N.A., Wang, P., Schaechter, A., Howard, A., Zareba, W., et al. (2006) Preserved Heart Rate Variability Identifies Low-Risk Patients with Nonischemic Dilated Cardiomyopathy: Results from the DEFINITE Trial. Heart Rhythm, 3, 281-286. http://dx.doi.org/10.1016/j.hrthm.2005.11.028

[20] Simon, T., Becker, R., Voss, F., Bikou, O., Hauck, M., Licka, M., Katus, H.A. and Bauer, A. (2008) Elevated B-Type Natriuretic Peptide Levels in Patients with Nonischemic Cardiomyopathy Predict Occurrence of Arrhythmic Events. Clinical Research in Cardiology, 97, 306-309. http://dx.doi.org/10.1007/s00392-007-0629-2

[21] Tuomainen, P., Hartikainen, J., Vanninen, E. and Peuhkurinen, K. (2005) Warm-Up Phenomenon and Cardiac Autonomic Control in Patients with Coronary Artery Disease. Life Sciences, 76, 2147-2158. http://dx.doi.org/10.1016/j.lfs.2004.06.033

[22] Tuomainen, P., Peuhkurinen, K., Kettunen, R. and Rauramaa, R. (2005) Regular Physical Exercise, Heart Rate Variability and Turbulence in a 6-Year Randomized Controlled Trial in Middle-Aged Men: The DNASCO Study. Life Sciences, 77, 2723-2734. http://dx.doi.org/10.1016/j.lfs.2005.05.023

[23] Schmidt, G., Malik, M., Barthel, P., Schneider, R., Ulm, K., Rolnitzky, L., et al. (1999) Heart-Rate Turbulence after Ventricular Premature Beats as a Predictor of Mortality after Acute Myocardial Infarction. The Lancet, 353, 1390-1396. http://dx.doi.org/10.1016/S0140-6736(98)08428-1

[24] Tuomainen, P.O., Magga, J., Timonen, P., Miettinen, K., Kurttila, M., Vanninen, E., Laitinen, T., Timonen, K., Punnonen, K., Parviainen, I., Uusaro, A., Vuolteenaho, O., Kivikko, M. and Peuhkurinen, K. (2013) Intermittent Levosimendan Treatment in Patients with Severe Congestive Heart Failure. Clinical Research in Cardiology, 102, 485-493. http://dx.doi.org/10.1007/s00392-013-0554-5

[25] Tuomainen, P.O., Magga, J., Fedacko, J., Kärkkäinen, S., Miettinen, K., Vanninen, E., Kuusisto, J. and Peuhkurinen, K.J. (2014) Idiopathic Dilated Cardiomyopathy and Chromic Atrial Fibrillation. Clinical Physiology and Functional Imaging, 34, 133-137. http://dx.doi.org/10.1111/cpf.12075

[26] La Rovere, M., Pinna, G., Maestri, R., Barlera, S., Bernardinangeli, M., Veniani, M., et al. (2012) Autonomic Markers and Cardiovascular and Arrhythmic Events in Heart Failure Patients: Still a Place in Prognostication? Data from the GISSI-HF Trial. European Journal of Heart Failure, 14, 1410-1419. http://dx.doi.org/10.1093/eurjhf/hfs126

[27] Baldasseroni, S., Opasich, C., Gorini, M., Lucci, D., Marchionni, N., Marini, M., et al. (2002) Left Bundle-Branch Block Is Associated with Increased 1-Year Sudden and Total Mortality Rate in 5517 Outpatients with Congestive Heart Failure: A Report from the Italian Network on Congestive Heart Failure. American Heart Journal, 143, 398-405. http://dx.doi.org/10.1067/mhj.2002.121264

[28] Hofmann, T., Meinertz, T., Kasper, W., Geibel, A., Zehender, M., Hohnloser, S., Stienen, U., Treese, N. and Just, H. (1988) Mode of Death in Idiopathic Dilated Cardiomyopathy: A Multivariate Analysis of Prognostic Determinants. American Heart Journal, 116, 1455-1463. http://dx.doi.org/10.1016/0002-8703(88)90728-4

[29] Ahmad, S., Otaal, P.S., Rai, T.S., Bahl, A., Saikia, U.N., Manoj, R.K., Thungapathra, M., Talwar, K.K. and Khullar, M. (2009) Circulating Proinflammatory Cytokines and N-Terminal Pro-Brain Natriuretic Peptide Significantly Decrease with Recovery of Left Ventricular Function in Patients with Dilated Cardiomyopathy. Molecular and Cellular Biochemistry, 324, 139-145. http://dx.doi.org/10.1007/s11010-008-9992-1

[30] Gulati, A., Ismail, T.F., Jabbour, A., Ismail, N.A., Morarji, K., Ali, A., Raza, S., Khwaja, J., et al. (2013) Clinical Utility and Prognostic Value of Left Atrial Volume Assessment by Cardiovascular Magnetic Resonance in Non-Ischaemic Dilated Cardiomyopathy. European Journal of Heart Failure, 15, 660-670. http://dx.doi.org/10.1093/eurjhf/hft019

[31] Sugrue, D.D., Rodeheffer, R.J., Codd, M.B., Ballard, D.J., Fuster, V. and Gersh, B.J. (1992) The Clinical Course of Idiopathic Dilated Cardiomyopathy: A Population-Based Study. Annals of Internal Medicine, 117, 117-123. http://dx.doi.org/10.7326/0003-4819-117-2-117

[32] Schefer, T., Wolber, T., Binggeli, C., Holzmeister, J., Brunckhorst, C. and Duru, F. (2008) Long-Term Predictors of Mortality in ICD Patients with Non-Ischaemic Cardiac Disease: Impact of Renal Function. Europace, 10, 1052-1059. http://dx.doi.org/10.1093/europace/eun186

[33] Grimm, W., Hoffmann, J., Menz, V., Luck, K. and Maisch, B. (1998) Programmed Ventricular Stimulation for Arrhythmia Risk Prediction in Patients with Idiopathic Dilated Cardiomyopathy and Nonsustained Ventricular Tachycardia. Journal of the American College of Cardiology, 32, 739-745. http://dx.doi.org/10.1016/S0735-1097(98)00306-4

[34] Lau, E.W., Griffith, M.J., Pathmanathan, R.K., Ng, G.A., Clune, M.M., Cooper, J., et al. (2004) The Midlands Trial of Empirical Amiodarone versus Electrophysiology-Guided Interventions and Implantable Cardioverter-Defibrillators (MAVERIC): A Multi-Centre Prospective Randomised Clinical Trial on the Secondary Prevention of Sudden Cardiac Death. Europace, 6, 257-266. http://dx.doi.org/10.1016/j.eupc.2004.03.009

[35] European Society of Cardiology, Heart Failure Association of the ESC (HFA), European Society of Intensive Care 
Medicine (ESICM), Dickstein, K., Cohen-Solal, A., Filippatos, G., et al. (2008) ESC Guidelines for the Diagnosis and Treatment of Acute and Chronic Heart Failure 2008: The Task Force for the Diagnosis and Treatment of Acute and Chronic Heart Failure 2008 of the European Society of Cardiology. Developed in Collaboration with the Heart Failure Association of the ESC (HFA) and Endorsed by the European Society of Intensive Care Medicine (ESICM). European Journal of Heart Failure, 10, 933-989.

[36] Binkley, P.F., Haas, G.J., Starling, R.C., Nunziata, E., Hatton, P.A., Leier, C.V., et al. (1993) Sustained Augmentation of Parasympathetic Tone with Angiotensin-Converting Enzyme Inhibition in Patients with Congestive Heart Failure. Journal of the American College of Cardiology, 21, 655-661. http://dx.doi.org/10.1016/0735-1097(93)90098-L

[37] Niemela, M.J., Airaksinen, K.E. and Huikuri, H.V. (1994) Effect of Beta-Blockade on Heart Rate Variability in Patients with Coronary Artery Disease. Journal of the American College of Cardiology, 23, 1370-1377. http://dx.doi.org/10.1016/0735-1097(94)90379-4

[38] Wilkins, M.R., Redondo, J. and Brown, L.A. (1997) The Natriuretic-Peptide Family. The Lancet, 349, 1307-1310. http://dx.doi.org/10.1016/S0140-6736(96)07424-7

[39] Vanderheyden, M., Goethals, M., Verstreken, S., De Bruyne, B., Muller, K., Van Schuerbeeck, E., et al. (2004) Wall Stress Modulates Brain Natriuretic Peptide Production in Pressure Overload Cardiomyopathy. Journal of the American College of Cardiology, 44, 2349-2354. http://dx.doi.org/10.1016/j.jacc.2004.09.038

[40] Iwanaga, Y., Nishi, I., Furuichi, S., Noguchi, T., Sase, K., Kihara, Y., et al. (2006) B-Type Natriuretic Peptide Strongly Reflects Diastolic Wall Stress in Patients with Chronic Heart Failure: Comparison between Systolic and Diastolic Heart Failure. Journal of the American College of Cardiology, 47, 742-748. http://dx.doi.org/10.1016/j.jacc.2005.11.030

[41] Adamson, P.B., Kleckner, K.J., Van Hout, W.L., Srinivasan, S. and Abraham, W.T. (2003) Cardiac Resynchronization Therapy Improves Heart Rate Variability in Patients with Symptomatic Heart Failure. Circulation, 108, $266-269$. http://dx.doi.org/10.1161/01.CIR.0000083368.75831.7A

[42] Gademan, M.G., van Bommel, R.J., Ypenburg, C., Haest, J.C., Schalij, M.J., van der Wall, E.E., et al. (2008) Biventricular Pacing in Chronic Heart Failure Acutely Facilitates the Arterial Baroreflex. AJP: Heart and Circulatory Physiology, 295, H755-H760. http://dx.doi.org/10.1152/ajpheart.00170.2008

[43] Machado, C.R., Camargos, E.R., Guerra, L.B. and Moreira, M.C. (2000) Cardiac Autonomic Denervation in Congestive Heart Failure: Comparison of Chagas' Heart Disease with Other Dilated Cardiomyopathy. Human Pathology, 31, 3-10. http://dx.doi.org/10.1016/S0046-8177(00)80191-4

[44] Rutschow, S., Li, J., Schultheiss, H.P. and Pauschinger, M. (2006) Myocardial Proteases and Matrix Remodeling in Inflammatory Heart Disease. Cardiovascular Research, 69, 646-656. http://dx.doi.org/10.1016/j.cardiores.2005.12.009

[45] Miettinen, K.H., Eriksson, S., Magga, J., Tuomainen, P., Kuusisto, J., Vanninen, E.J., et al. (2008) Clinical Significance of Troponin I Efflux and Troponin Autoantibodies in Patients with Dilated Cardiomyopathy. Journal of Cardiac Failure, 14, 481-488. http://dx.doi.org/10.1016/j.cardfail.2008.02.009

[46] Francis, J., Zhang, Z.H., Weiss, R.M. and Felder, R.B. (2004) Neural Regulation of the Proinflammatory Cytokine Response to Acute Myocardial Infarction. AJP: Heart and Circulatory Physiology, 287, H791-H797. http://dx.doi.org/10.1152/ajpheart.00099.2004 